Proyecciones Journal of Mathematics

Vol. 33, $\mathrm{N}^{o}$ 4, pp. 369-388, December 2014.

Universidad Católica del Norte

Antofagasta - Chile

\title{
L(1,1)-Labeling of Direct Product of any Path and Cycle
}

\author{
Deborah Olayide Ajayi \\ University of Ibadan, Nigeria \\ and \\ Charles Adefokun \\ Crawford University, Nigeria \\ Received: November 2013. Accepted: September 2014
}

\begin{abstract}
Suppose that $[n]=\{0,1,2, \ldots, n\}$ is a set of non-negative integers and $h, k \in[n]$. The $L(h, k)$-labeling of graph $G$ is the function $l$ : $V(G) \rightarrow[n]$ such that $|l(u)-l(v)| \geq h$ if the distance $d(u, v)$ between $u$ and $v$ is 1 and $|l(u)-l(v)| \geq k$ if $d(u, v)=2$. Let $L(V(G))=$ $\{l(v): v \in V(G)\}$ and let $p$ be the maximum value of $L(V(G))$. Then $p$ is called $\lambda_{h}^{k}$-number of $G$ if $p$ is the least possible member of $[n]$ such that $G$ maintains an $L(h, k)$-labeling. In this paper, we establish $\lambda_{1}^{1}-$ numbers of $P_{m} \times P_{n}$ and $P_{m} \times C_{n}$ graphs for all $m, n \geq 2$.
\end{abstract}

Keywords : L(1,1)-labeling, D-2 Coloring, Direct Product of Graphs, Cross Product of Graphs, Path and Cycle.

Mathematics Subject Classification : Primary: $05 C 78$. 


\section{Introduction}

Let $l: V(G) \rightarrow[n]=\{0,1,2, \cdots, n\}$ be a non negative function on the vertex set $V(G)$ of $G$. Given any two fixed non-negative integers $h, k$, the $L(h, k)$-labeling of $G$ is defined such that for any edge $u v \in E(G)$, $|l(u)-l(v)| \geq h$ and if $d(u, v)=2, u, v \in V(G)$, then $|l(u)-l(v)| \geq k$. The aim of $L(h, k)$-labeling is to obtain the smallest non negative integer $\lambda_{h}^{k}(G)$, such that there exists an $L(h, k)$-labeling of $G$ with no $l(v) \in L(V(G))$ greater than $\lambda_{h}^{k}(G)$, where $L(V(G))$ is the set of all labels on $V(G)$.

In [13], Griggs and Yeh introduced the $l(h, k)$-labelling and particularly showed that any graph $G$ with maximum degree $\Delta>1$ has $\lambda_{2}^{1}(G) \leq \Delta^{2}+$ $2 \Delta$ and went further to put forward a conjecture that $\lambda_{2}^{1}(G) \leq \Delta^{2}$. Chang and Kuo, in [5] improved on Griggs and Yeh's bound by showing that $\lambda_{2}^{1}(G) \leq \Delta(\Delta+1)$, Kral' and Skrekovski [16] went another step showing that $\lambda_{2}^{1}(G) \leq \Delta(\Delta+1)-1$ while Goncalves in [11] proved that $\lambda_{2}^{1}(G) \leq$ $\Delta(\Delta+1)-2$. The interest in the Griggs-Yeh conjecture and in improving on the existing bounds have inspired a lot of work in the direction of $L(h, k)$ labeling, mostly on $h=2, k=1$. (See [5][6][10][12][18].) (An extensive review of all known results on $L(h, k)$-labeling can be seen in [3].) It is obvious that $L(2,1)$-labeling is an $L(1,1)$-labeling, therefore results on $L(2,1)$-labeling provide upper bound for $L(1,1)$-labeling of graphs and

$$
\lambda_{2}^{1}(G)+1 \geq \lambda_{1}^{1}(G)+1=\lambda\left(G^{2}\right)
$$

where $\lambda\left(G^{2}\right)$ is the chromatic number of the square of $G$.

Finally, Georges and Mauro [8] obtained various results for the $L(h, k)$-number for path $P_{n}$ and cycles $C_{n}$. Particularly among other results, they showed that $\lambda_{h}^{k}\left(P_{n}\right)$ is either $0, h, h+k, h+2 k$, or $2 h$.

Suppose that $G$ and $H$ are graphs. The Cartesian product and the direct product of $G$ and $H, G \square H$ and $G \times H$ respectively, have vertex set $V(G) \times V(H)$, while the edge sets are

$E(G \square H)=\left\{\left(\left(x_{1}, x_{2}\right),\left(y_{1}, y_{2}\right)\right):\left(x_{1}, y_{1}\right) \in E(G)\right.$ and $x_{2}=y_{2}$ or $\left(x_{2}, y_{2}\right) \in E(H)$ and $\left.x_{1}=y_{1}\right\}$ and

$E(G \times H)=\left\{\left(\left(x_{1}, x_{2}\right),\left(y_{1}, y_{2}\right)\right):\left(x_{1}, y_{1}\right) \in E(G) \quad\right.$ and $\left.\left(x_{2}, y_{2}\right) \in E(H)\right\}$ respectively.

The $L(h, k)$-labeling of the Cartesian product $G \square H$ has been extensively investigated with $\lambda_{h}^{k}(G \square H)$ obtained for various types of graphs $G$ and $H$, while numerous upper and lower bounds have been suggested (see [8][7][16][18][20][22]). Most of the work on $L(h, k)$ labeling consider $h=2$ and $k=1$; although Chiang and Yan in [7] and Georges and Mauro in [10] 
worked on the $L(1,1)$ labeling of Cartesian products of paths and cycles and Sopena and $\mathrm{Wu}$ in [20] worked on Cartesian products of cycles. In case of direct product graphs, Jha et al [15], established $\lambda_{2}^{1}\left(C_{m} \times C_{n}\right)$ for some values of $m$ and $n$.

In this paper, we determine $\lambda_{1}^{1}\left(P_{m} \times P_{n}\right)$ and $\lambda_{1}^{1}\left(P_{m} \times C_{n}\right)$ where $P_{m}$ and $P_{n}$ are paths of length $m-1$ and $n-1$ respectively and $C_{n}$ is a cycle of length $n$ for all $m, n \geq 2$. We also deduce $\lambda_{1}^{1}\left(C_{m} \times C_{n}\right)$ for $m, n \equiv 0 \bmod 5$. Thus, we extend the results in [10] and [7] to direct product graphs among other results.

\section{Preliminaries}

The following results and definitions are necessary.

Let $m$ be a non-negative integer. $P_{m}=u_{0} u_{1} u_{2} \ldots u_{m-1}$ is a path of length $m-1$, where $u_{i} \in V\left(P_{m}\right)$, for all $i \in[m-1] ; C_{m}=u_{0} u_{1} u_{2} \ldots u_{m-1} u_{0}$ is a cycle of length $m$, where $u_{i} \in V\left(C_{m}\right)$, for all $i \in[m-1]$. Let $v \in V(G)$, we denote by $l(v)$ the label on $v$ and let $U \subseteq V(G)$. Then $L(U)$ is a set of labels on $U$.

Suppose $P_{m} \times P_{n}$ is a direct product paths and $G^{\prime}$ is a component of $P_{m} \times P_{n}$. Then

$U_{j}=\left\{u_{i} v_{j}\right\} \subset V\left(G^{\prime}\right)$, for some $j \in[n-1]$, and for all $i \in[(m-1)(\epsilon)]$ or for all $i \in[(m-1)(o)]$.

$V_{i}=\left\{u_{i} v_{j}\right\} \subset V\left(G^{\prime}\right)$, for some $i \in[m-1]$, and for all $j \in[(n-1)(\epsilon)]$ or for all $j \in[(n-1)(o)]$.

Theorem 2.1. [22] Graph $G \times H$ is connected if and only if $G$ and $H$ are connected and at least one of $G$ and $H$ is non-bipartite.

\section{Remark 2.2.}

(i) Since $P_{m}$ is bipartite for all $m \geq 2$, then for $P_{m} \times P_{n}$, there exist $G_{1} \subset P_{m} \times P_{n}$ and $G_{2} \subset P_{m} \times P_{n}$ such that $G_{1}$ and $G_{2}$ are components of $P_{m} \times P_{n}$.

(ii) From Theorem 2.1 and the Remark above, it is clear that $P_{m} \times P_{n}$ is not a connected graph. Suppose $P_{m}=u_{0} u_{1} u_{2} \ldots u_{m-1}$ and $P_{n}=$ $v_{0} v_{1} v_{2} \ldots v_{n-1}$, then

$V\left(G_{1}\right)=\left\{u_{i}, v_{j}: i \in[(m-1)(\epsilon)], j \in[(n-1)(\epsilon)]\right.$

or $i \in[(m-1)(o)] ; j \in(n-1)[o]$

$V\left(G_{2}\right)=\left\{u_{i}, v_{j}: i \in[(m-1)(\epsilon)], j \in[(n-1)(o)]\right.$ 
or $i \in[(m-1)(o)] ; j \in(n-1)[\epsilon]$.

(iii) Suppose $G$ is a graph such that $G=G^{\prime} \cup G^{\prime \prime}$, where $G^{\prime}, G^{\prime \prime}$ are components of $\mathrm{G}$, then, $\lambda_{1}^{1}(G)=\max \left\{\lambda_{1}^{1}\left(G^{\prime}\right), \lambda_{1}^{1}\left(G^{\prime \prime}\right)\right\}$.

(iv) For a direct product graph, $P_{m} \times P_{2}, m \geq 2$, its components $G_{1}$ and $G_{2}$ are paths $P_{m}^{\prime}$ and $P_{m}^{\prime \prime}$ respectively such that $P_{m}^{\prime}=u_{0} v_{0} u_{1} v_{1} u_{2} v_{0} \ldots u_{m-1} v_{1}\left(u_{m-1} v_{0}\right)$ (if $m$ is even) and $P_{m}^{\prime \prime}=u_{0} v_{1} u_{1} v_{0} u_{2} v_{1} \ldots u_{m-1} v_{0}\left(u_{m-1} v_{1}\right)$ (if $m$ is odd).

The following are known results for $L(1,1)$-labeling of paths, cycles and $L(h, k)$-labeling of stars, $k \leq h$.

Lemma 2.3. [1] Let $P_{m}$ be a path of length $m-1$. $\lambda_{1}^{1}\left(P_{m}\right)=1$, for $m=2$ and $\lambda_{1}^{1}\left(P_{m}\right)=2$ for all $m \geq 3$.

Lemma 2.4. [1] Let $C_{m}$ be cycle of length $m$. Then $\lambda_{1}^{1}\left(C_{m}\right)=2$ for $m \equiv 0 \bmod 3$ and $\lambda_{1}^{1}\left(C_{m}\right)=3$ for $m \not \equiv 0 \bmod 3$.

The following result presents a general $\lambda_{h}^{k}$-value for stars for $k \leq h$.

Lemma 2.5. [4] Let $K_{1, \Delta}$ be a star of order $\Delta+1$. Then, $\lambda_{h}^{k}\left(K_{1, \Delta}\right)=$ $(\Delta-1) k+h$ if $h \geq k$.

Henceforth we refer to direct product graph as product graph.

3. $L(1,1)$-Labeling of $P_{m} \times P_{n}$

Proposition 3.1. $\lambda_{1}^{1}\left(P_{2} \times P_{2}\right)=1$.

Proof. Clearly, $G$ consists of connected components $P_{2}^{\prime}$ and $P_{2}^{\prime \prime}$. By Lemma 2.3, $\lambda_{1}^{1}\left(P_{2}^{\prime}\right)=\lambda_{1}^{1}\left(P_{2}^{\prime \prime}\right)=1$.

We extend the graph in Theorem 3.1 to $m \geq 3$.

Proposition 3.2. For $m \geq 3, \lambda_{1}^{1}\left(P_{m} \times P_{2}\right)=2$. 
Proof. $\quad P_{m} \times P_{2}$ consists of two connected components $P_{m}^{\prime}$ and $P_{m}^{\prime \prime}$. By Lemma 2.3, $\lambda_{1}^{1}\left(P_{m}^{\prime}\right)=\lambda_{1}^{1}\left(P_{m}^{\prime \prime}\right)=2$ and the result follows from Remark 2.2 (iii).

The next results establish $\lambda_{1}^{1}\left(P_{m} \times P_{n}\right), m, n \geq 3$.

Lemma 3.3. Let $u_{i} v_{j} \in P_{m} \times P_{n}, n, m \geq 3$, Suppose $d_{u_{i}}=d_{v_{j}}=2$ then $d_{u_{i} v_{j}}=4$.

Proof. Let $u_{i-1} u_{i} u_{i+1}=P_{3}^{\prime}, P_{3}^{\prime} \subseteq P_{m}, m \geq 3$ and let $v_{j-1} v_{j} v_{j+1}=$ $P_{3}^{\prime \prime}, P_{3}^{\prime \prime} \subseteq P_{n}, n \geq 3$. By the definition of direct product of graphs, $V\left(P_{m}^{\prime} \times P_{n}^{\prime \prime}\right)=$

$\left\{u_{i-1} v_{j-1}, u_{i-1} v_{j}, u_{i-1} v_{j+1}, u_{i} v_{j-1}, u_{i} v_{j}, u_{i} v_{j+1}, u_{i+1} v_{j-1}, u_{1+1} v_{j}, u_{i+1} v_{j+1}\right\}$ $\subseteq V\left(P_{m} \times P_{n}\right)$. Since $d_{u_{i}}=d_{v_{j}}=2$, then by the definition of direct product of graphs, $u_{i} v_{j} \in V\left(P_{3}^{\prime} \times P_{3}^{\prime \prime}\right)$ is adjacent to all the members of $\left\{u_{i-1} v_{j-1}, u_{i+1} v_{j-1}, u_{i+1} v_{j+1}, u_{i-1} v_{j+1}\right\}$. Thus, $d_{u_{i} v_{j}}=4$.

Proposition 3.4. Suppose $m, n \geq 3$. Then $\lambda_{1}^{1}\left(P_{m} \times P_{n}\right)=4$ for all $m, n \geq 3$.

Proof. Let $G_{1}$ be a connected component of $P_{m} \times P_{n}$. By Lemma 3.3, there exists a star $K_{1,4} \subseteq G_{1}$. By Lemma 2.5, $\lambda_{1}^{1}\left(K_{1,4}\right)=4$ and thus, $\lambda_{1}^{1}\left(P_{m} \times P_{n}\right) \geq 4$. Let $u_{i} v_{j} \in V\left(P_{m} \times P_{n}\right)$. For all $u_{i} v_{j} \in V\left(P_{m} \times P_{n}\right)$, $l\left(u_{i} v_{j}\right)=\left\lfloor\frac{i+3 j}{2}\right\rfloor \bmod 5$. Thus $\lambda_{1}^{1}\left(P_{m} \times P_{n}\right) \leq 4$ and then the equality follows.

Remark 3.5. By using $l\left(u_{i} v_{j}\right)=\left\lfloor\frac{i+3 j}{2}\right\rfloor \bmod 5$ as in the proof of Proposition 3.4, given both connected components of $P_{m} \times P_{n}$, for all $i \in[m(\epsilon)]$, then $1\left(\mathrm{u}_{i} v_{10}\right)=l\left(u_{i} v_{0}\right)$. Furthermore, for all $u_{i} v_{1} \in U_{1}, i \in\{3,5,7\} l\left(u_{i} v_{1}\right) \notin L\left(u_{i-2} v_{9}, u_{i} v_{9}, u_{i+2} v_{9}\right)$,

$\left\{u_{i-2} v_{9}, u_{i} v_{9}, u_{i+2} v_{9}\right\} \subset U_{9}$. We also notice that $l\left(u_{1} v_{1}\right) \notin L\left(u_{1} v_{9}, u_{3} v_{9}, u_{9} v_{9}\right)$, while $l\left(u_{9} v_{1}\right) \notin L\left(u_{1} v_{9}, u_{7} v_{9}, u_{9} v_{9}\right)$. Also, for all $u_{1} v_{j} \in V_{1}, j \in\{3,5,7\}, l\left(u_{1} v_{j}\right) \notin L\left(u_{9} v_{j-2}, u_{9} v_{j}, u_{9} v_{j+2}\right)$, $\left\{u_{9} v_{j-2}, u_{9} v_{j}, u_{9} v_{j+2}\right\} \subset V_{9}$ and $l\left(u_{1} v_{1}\right) \notin L\left(u_{9} v_{1}, u_{9} v_{3}, u_{9} v_{9}\right)$, while $l\left(u_{1} v_{9}\right) \notin$ $L\left(u_{9} v_{1}, u_{9} v_{7}, u_{9} v_{9}\right)$.

The implication of Remark 3.5 is expressed in the following results.

Corollary 3.6. Let $C_{m}$ be a cycle of length $m$, then, $\lambda_{1}^{1}\left(C_{10} \times C_{10}\right)=4$.

Corollary 3.7. For all $m, n \equiv 0 \bmod 5, \lambda_{1}^{1}\left(C_{m} \times C_{n}\right)=4$. 


\section{4. $L(1,1)$-Labeling of $P_{m} \times C_{m}$}

Lemma 4.1. Let $G=P_{m} \times P_{n}$, where $n \geq 4$. Suppose that $\alpha_{k} \in$ [4], such that for some $v_{i} \in V(G), l\left(v_{i}\right)=\alpha_{k}, v_{j} \in V(G)$ is the closest vertex in $V(G)$ to $v_{i}, i \neq j$ such that $l\left(v_{j}\right)=\alpha_{k}$. Then $3 \leq d\left(v_{i}, v_{j}\right) \leq 4$.

Proof. That $3 \leq d\left(v_{i}, v_{j}\right)$ follows directly from the definition of $L(1,1)$ labeling. Next, we show that $d\left(v_{i}, v_{j}\right) \leq 4$. Let $S_{n}$ be a star of order $n+1$. Clearly, $\operatorname{diam}\left(S_{n}\right)=2$. Now, suppose that for two stars $S_{4}^{\prime} \subset G$ and $S_{4}^{\prime \prime} \subset G$, there exits some vertex $u_{i}$ such that $u_{i} \in V\left(S_{n}^{\prime}\right)$ and also $u_{i} \in V\left(S_{n}^{\prime \prime}\right)$, making $S_{n}^{\prime}$ and $S_{n}^{\prime \prime}$ to be neighbors. Then, $\operatorname{diam}(H)=4$, where $S_{4}^{\prime} \cup S_{4}^{\prime \prime}=H \subset G$. Now, suppose $d\left(v_{i}, v_{j}\right)>4$. Let $v_{i} \in V\left(S_{4}^{\prime}\right)$ such that $l\left(v_{i}\right)=\alpha_{k}$. Also, let $L\left(S_{4}^{\prime}\right)=[4]$. Then, $\alpha_{k} \neq l\left(v_{k}\right)$ for all $v \in V\left(S_{4}^{\prime \prime}\right)$ since $d\left(u_{i}, v_{j}\right) \geq 4$. Thus, there exits some $\alpha_{j} \notin[4]$ such that $\alpha_{j} \in L\left(S_{4}^{\prime \prime}\right)$. Then, $\lambda_{1}^{1}(H) \geq 5$, and consequently, $\lambda_{1}^{1}(G) \geq 5$. This is a contradiction.

Lemma 4.2. Let $v_{i}, v_{j} \in V(G)$ be two center vertices of stars $S_{4}^{\prime}, S_{4}^{\prime \prime} \subset G$ respectively, and that $d\left(v_{i}, v_{j}\right)=4$ if $\alpha_{i}=l\left(v_{i}\right)$ and $\alpha_{j}=l\left(v_{j}\right), \alpha_{i}, \alpha_{j} \in$ [4], then $\alpha_{i} \neq \alpha_{j}$.

Proof. Suppose on the contrary that $v_{i}, v_{j}$ are respective centers of $S_{4}^{\prime}, S_{4}^{\prime \prime}$ such that $d\left(v_{i}, v_{j}\right)=4$ and $\alpha_{i}=\alpha_{j}$. There exists a star $S_{4}^{\prime \prime \prime} \subset G$ with $V\left(S_{4}^{\prime \prime \prime}\right)=u_{q} v_{r}, u_{q+2} v_{r}, u_{q+1} v_{r+1}, u_{q} v_{r+2}, u_{q+2} v_{r+2}$, where $0 \leq q, q+$ $2 \leq m$ and $r \leq 2, r+2 \leq n-3$, such that $v_{i}=u_{q+1} v_{r-1}$ and $v_{j}=$ $u_{q+1} v_{r+3}$. Therefore $v_{i}$ is adjacent to $u_{q} v_{r}$ and $u_{q+2} v_{r}$ and $d\left(v_{i}, u_{q+1} v_{r+1}\right)=$ 2. Likewise, $v_{j}$ is adjacent to both $u_{q} v_{r+2}, u_{q+2} v_{r+2}$ and $d\left(v_{j}, u_{q+1} v_{r+1}\right)=$ 2. Thus there exists no vertex $v_{l} \in V\left(S^{\prime \prime \prime}\right)$ such that $l\left(v_{l}\right)=\alpha_{i} \in[4]$. This contradicts the fact that $\lambda_{1}^{1}(G) \leq 4$, for all $m, n \geq 2$.

Lemma 4.3. Let $G^{\prime} \subset G$ with

$V\left(G^{\prime}\right)=\left\{u_{q} v_{r}, u_{q+2} v_{r}, u_{q+1} v_{r+1}, u_{q} v_{r+2}, u_{q+2} v_{r+2}, u_{q+1} v_{r+3}, u_{q} v_{r+4}, u_{q+2} v_{r+4}\right\}$, $q, r \geq 0$. Suppose that $l\left(u_{q} v_{r}\right), l\left(u_{q+2} v_{r}\right)$ are $\alpha_{0}, \alpha_{1}$ respectively, then $l\left(u_{q} v_{r+4}\right), l\left(u_{q+2} v_{r+4}\right)$ are both neither $\alpha_{0}$ nor $\alpha_{1}$.

Proof. The vertex set $\left\{u_{q} v_{r}, u_{q+2} v_{r}, u_{q+1} v_{r+1}, u_{q} v_{r+2}, u_{q+2} v_{r+2}\right\} \subset V^{\prime}\left(G^{\prime}\right)$ induces a star $S_{4} \subset G$. Since $\lambda_{1}^{1}\left(S_{4}\right)=4$, we have $l\left(u_{q+1} v_{r+1}\right)=\alpha_{2}, l\left(u_{q} v_{r+2}\right)=\alpha_{3}, l\left(u_{q+2} v_{r+2}\right)=\alpha_{4}$. Set 
$\left\{u_{q} v_{r+2}, u_{q+2} v_{r+2}, u_{q+1} v_{r+3}, u_{q} v_{r+4}, u_{q+2} v_{r+4}\right\} \subset V\left(G^{\prime}\right)$ induces another star $S_{4}^{\prime} \subset G^{\prime}$. Clearly, $S_{4}$ and $S_{4}^{\prime}$ are adjacent and $S_{4} \cup S_{4}^{\prime}=G^{\prime}$ Now, suppose $l\left(u_{q} v_{r+4}\right)=\alpha_{0}, l\left(u_{q+2} v_{r+4}\right)=\alpha_{1}$, or vice versa without the loss of generality. Since $l\left(u_{q} v_{r+2}\right)=\alpha_{3}$, and $l\left(u_{q+2} v_{r+2}\right)=\alpha_{3}$ from the labeling on $S_{4}$, the only label left in [4] for $u_{q+1} v_{r+3}$ is $\alpha_{2}$. This however is a contradiction since $d\left(u_{q+1} v_{r+1}, u_{q+1} v_{r+3}\right)=2$.

\section{Remark 4.4.}

(i) By theorem 2.1, $P_{m} \times C_{n}$ is connected if $n$ is odd and not connected if $n$ is even. This is because when $n$ is odd, cycle $C_{n}$ is non bipartite and when $n$ is even, $C_{n}$ is bipartite. Now, Let $P_{m} \times C_{n}=G=G_{1} \cup G_{2}$, where $n$ is even. Then

$V\left(G_{1}\right)=\left\{\left(u_{i}, v_{j}\right): i \in[(m-1)(\epsilon)], j \in[n(\epsilon)]\right.$ or $\left.i \in[(m-1)(o)], j \in[n(o)]\right\}$ and

$V\left(G_{2}\right)=\left\{\left(u_{i}, v_{j}\right): i \in[(m-1)(\epsilon)], j \in[n(o)]\right.$ or $\left.i \in[(m-1)(o)], j \in[n(\epsilon)]\right\}$.

(ii) $G_{1}$ and $G_{2}$ above are isomorphic since $C_{n}$ is a cycle and they are both components of $G$.

(iii) Suppose $G=P_{m} \times C_{n}, n$ odd. Then $G$ is equivalent to $G^{\prime}$, where $G^{\prime}$ is one of the two components of $P_{m} \times C_{2 n}$.

(iv) $G^{\prime}$ above is equivalent to the connected component of $P_{m} \times P_{2 n+1}$ such that $u_{i} v_{0}$ coincides with $u_{i} v_{2 n}$, for all $i \in[(m-1)(\epsilon)]$ or for all $i \in[(m-1)(o)]$.

Lemma 4.5. $[2] \lambda_{1}^{1}\left(C_{m}\right)= \begin{cases}2 & \text { if } m \equiv 0 \bmod 3 \\ 3 & m \neq \equiv 0 \bmod 3 ; m \neq 5 \\ 4 & m=5\end{cases}$

Theorem 4.6. $\lambda_{1}^{1}\left(P_{2} \times C_{m}\right)= \begin{cases}2 & \text { if } m \equiv 0 \bmod 3 \\ 3 & \text { otherwise. }\end{cases}$

Proof. By Remark 4.4 (iii), if $m$ is odd, then $P_{2} \times C_{m} \equiv C_{2 m}$. If $m$ is even, then $P_{2} \times C_{m}$ is a union of $m$-cycles, $C_{m}^{\prime}$ and $C_{m}^{\prime \prime}$ are $m-$ cycles which are its components. By Lemma 4.5, for $m$ odd, $\lambda_{1}^{1}\left(P_{2} \times C_{m}\right)=$ $\lambda_{1}^{1}\left(C_{2 m}\right)=q$, where $q=2$ for $2 m \equiv 0 \bmod 3$ and $q=3$ if otherwise. Also $\lambda_{1}^{1}\left(P_{2} \times C_{2}\right)=\lambda_{1}^{1}\left(C_{n}\right)=p$, where $p=2$ if $n \equiv 0 \bmod 3$ and $p=3$ otherwise. 
Theorem 4.7. For any $m \in \mathbf{N}, m \geq 3, \lambda_{1}^{1}\left(P_{m} \times C_{3}\right)=5$.

Proof. By Remarks 4.4 (iii) and (iv), and $P_{m} \times C_{3}$ is congruent to a connected component $G^{\prime}$ of $P_{m} \times P_{7}$ with $u_{i} v_{0} \equiv u_{i} v_{6}, u_{i} v_{0}, u_{i} v_{6} \in V\left(G^{\prime}\right)$. Thus, $L\left(u_{i} v_{0}\right)=L\left(u_{i} v_{6}\right)$ for all $i \in[(m-i)(\epsilon)]$. Now, let $G^{\prime \prime}$ be a subgraph of $G^{\prime}$ induced by the vertex subset

$\left\{u_{i} v_{0}, u_{i+2} v_{0}, u_{i+1} v_{1}, u_{i} v_{2}, u_{i+2} v_{2}, u_{i+1} v_{3}, u_{i} v_{4}, u_{i+2} v_{4}, u_{i+1} v_{5}, u_{i} v_{6}, u_{i+1} v_{6}\right\} \subseteq$ $V\left(G^{\prime}\right)$, for any $i \in[(m-1)(\epsilon)]$. Suppose $\lambda_{1}^{1}\left(G^{\prime}\right)=4$ and $\alpha_{0}, \alpha_{1}, \alpha_{2}, \alpha_{3}, \alpha_{4} \in$ [4]. Let $l\left(u_{i} v_{0}\right)=\alpha_{0}$ and $l\left(u_{i+2} v_{0}\right)=\alpha_{1}$. Then, $l\left(u_{i} v_{6}\right)=\alpha_{0}$ and $l\left(u_{i+2} v_{6}\right)=$ $\alpha_{1}$. Now, suppose $l\left(u_{i+1} v_{1}\right)=\alpha_{2}$. Since $d\left(u_{i+1} v_{1}, u_{i+1} v_{5}\right)=2$, then for some $\alpha_{k} \in[4], \alpha_{k}=l\left(u_{i+1} v_{5}\right) \neq \alpha_{2}$. In fact, $\alpha_{k} \notin\left\{\alpha_{0}, \alpha_{1}, \alpha_{2}\right\}$. Set $\alpha_{k}=\alpha_{3}$. The vertex subset $\left\{u_{i} v_{0}, u_{i+2} v_{0}, u_{i+1} v_{1}, u_{i} v_{2}, u_{i+2} v_{2}\right\} \subset V\left(G^{\prime \prime}\right)$ induces a star $S_{4} \subset G^{\prime}$ with center $u_{i+1} v_{1}$. Since $\lambda_{1}^{1}\left(S_{4}\right)=4$, if $l\left(u_{i} v_{2}\right)=\alpha_{3}$, then $l\left(u_{i+2} v_{2}\right)=\alpha_{4}$. Let $A$ and $B$ be vertex subsets of $V\left(G^{\prime}\right)$, such that $A=\left\{u_{i} v_{4}, u_{i+2} v_{4}\right\}$ and $B=\left\{u_{i} v_{2}, u_{i+2} v_{2}, u_{i+1} v_{5}, u_{1} v_{6}, u_{i+2} v_{6}\right\}$. Clearly, $d(u, v) \leq 2$ for all $u \in A$ and $v \in B$. Then, $l\left(u_{i} v_{4}\right), l\left(u_{i+2} v_{4}\right) \notin\left\{\alpha_{0}, \alpha_{1}, \alpha_{2}, \alpha_{3},\right\}$. Therefore, since $\lambda_{1}^{1}\left(S_{4}\right)=4, l\left(u_{i} v_{4}\right)=\alpha_{3}=l\left(u_{i+2} v_{4}\right)$. But $d\left(u_{i} v_{4}, u_{i+2} v_{4}\right)=$ 2. This a contradiction and hence, $\lambda_{1}^{1}\left(P_{m} \times C_{3}\right) \geq 5$.

Claim: Let $\alpha_{k} L\left(V_{i}\right)$, then $\alpha_{k} \notin V_{i+2}$, for $V_{i}, V_{i+2} \in V\left(G^{\prime}\right)$.

Reason: For all $v \in V_{i}, u \in V_{i+2}, d(u, v) \leq 2$.

Now, let $U_{i}=\left\{u_{i} v_{0}, u_{i} v_{2}, u_{1} v_{4}\right\}, U_{i+1}=\left\{u_{i+1} v_{1}, u_{i+1} v_{3}, u_{i+1} v_{5}\right\}, U_{i}, U_{i+1} \subset$ $V\left(G^{\prime \prime}\right) . l\left(u_{i} v_{j}\right)$ labels $u_{i+1}$ for all $v_{j}, u_{k}$ in $U_{i} U_{i+1}$ respectively where $|k-j|=$ 3 since $d\left(u_{i} v_{j}, u_{i} v_{k}\right)=3$. Therefore without loss of generality, we say $L\left(U_{i}\right)=L\left(U_{i+1}\right)=\left\{\alpha_{0}, \alpha_{1}, \alpha_{2}\right\} \subset[5]$. Likewise, let

$U_{i+2}=\left\{u_{i+2} v_{0}, u_{i+2} v_{2}, u_{i+2} v_{4}\right\}$ and

$U_{i+3}=\left\{u_{i+3} v_{1}, u_{i+3} v_{3}, u_{i+3} v_{5}\right\}, U_{i+2}, U_{i+3} \subset V\left(G^{\prime \prime}\right) . l\left(u_{i+2} v_{l}\right)$ labels $u_{i+3} v_{p}$ for all $v_{l}, v_{p}$ in $U_{i+2}, U_{i+3}$ respectively, where $|l-p|=3$. Thus $L\left(U_{i+2}\right)=$ $L\left(U_{i+3}\right)=\left\{\alpha_{3}, \alpha_{4}, \alpha_{5}\right\} \subset[5]$. Based on the last scheme, we have $L\left(U_{a}\right)=$ $L\left(U_{a+4}\right)$ for any $a \in[i, i+3]$, where $i \in[(m-1)(\epsilon)]$. Thus there exists a $5-L(1,1)$-labeling of $P_{m} \times C_{3}$ and thus $\lambda_{1}^{1}\left(P_{m} \times C_{3}\right) \leq 5$ and then the equality holds.

Corollary 4.8. If $m \geq 3$, then, $\lambda_{1}^{1}\left(P_{m} \times C_{6}\right)=5$.

Proof. Follows from Remark 4.4 (iii) and Theorem 4.7.

Theorem 4.9. If $m \geq 3$, then $\lambda_{1}^{1}\left(P_{m} \times C_{4}\right)=5$. 
Proof. From Remarks 4.4 (ii) and (iii), $P_{m} \times C_{4}=G_{1} \cup G_{2}$, where $G_{1}, G_{2}$ are isomorphic connected components of $P_{m} \times C_{4}$. Let $u_{i} v_{0}, u_{i} v_{4} \in V\left(G_{1}\right)$, say, for all $i \in[(m-1)(\epsilon)]$, such that $u_{i} v_{0} \equiv u_{i} v_{4}$ then by Remark 4.4 (iv), $G_{1}$ is equivalent to a connected component of $P_{m} \times P_{5}$. Now, let $G_{1}^{\prime} \subseteq G_{1}$ be a subgraph of $G_{1}$ with

$V\left(G_{1}^{\prime}\right)=\left\{u_{r} v_{0}, u_{r+2} v_{0}, u_{r+1} v_{1}, u_{r} v_{2}, u_{r+2} v_{2}, u_{r+1} v_{3}, u_{r} v_{4}, u_{r+2} v_{4}\right\}$, where $r \leq$ $m-4$. Obviously, $u_{r} v_{0} \equiv u_{r} v_{4}$ and $u_{r+2} v_{0} \equiv v_{4}$. Thus, $l\left(u-r v_{0}\right)=l\left(u_{r} v_{4}\right)=$ $\alpha_{i}$ and $l\left(u_{r+2} v_{0}\right)=l\left(u_{r+2} v_{4}\right)=\alpha_{j}, \alpha_{i}, \alpha_{j} \in[4]$. By Lemma 4.3, there exists a vertex $v \in V\left(G_{1}^{\prime}\right)$ such that $l(v) \notin[4]$. Thus $\lambda_{1}^{1}\left(G_{1}^{\prime}\right) \geq 5$ and therefore, $\lambda_{1}^{1}\left(G_{1}\right) \geq 5$ and finally, $\lambda_{1}^{1}\left(P_{m} \times C_{4}\right) \geq 5$. Now, for any pair $v_{a}, v_{b} \in V\left(G,,_{1}\right)$, $d\left(v_{a}, v_{b}\right) \leq 2$. Thus $L\left(V_{i}\right) \cap L\left(V_{i+1}\right)=\emptyset$ and $L\left(V_{i}\right) \cap L\left(V_{i+2}\right)=\emptyset$. However, $L\left(V_{i}\right)$ labels $L(V i+3)$ since $d\left(v_{a}, v_{c}\right)=3$ for all $v_{a} \in V_{i}$ and $v_{c} \in V_{i+3}$. Thus, $L\left(V_{i}\right)=L\left(V_{i+3 k}\right), L\left(V_{i+1}\right)=L\left(V_{i+4 k}\right)$ and $L\left(V_{i+2}\right)=L\left(V_{i+5 k}\right)$ for all $k \in \mathbf{N}$. since $\left|V\left(G_{1}^{\prime}\right)\right|=6$, then $\lambda_{1}^{1}\left(P_{m} \times C_{4}\right) \leq 5$ and therefore, the equality follows.

Theorem 4.10. If $m \geq 3$, then $\lambda_{1}^{1}\left(P_{m} \times C_{5}\right)=4$.

Proof. Clearly, $P_{m} \times C_{5} \equiv G_{1}$, where $G_{1}$ is a connected component of $P_{m} \times C_{10}$.

Therefore, $\lambda_{1}^{1}\left(P_{m} \times C_{5}\right) \leq \lambda_{1}^{1}\left(P_{m} \times C_{10}\right) \leq \lambda_{1}^{1}\left(C_{10 m^{\prime}} \times C_{10 n^{\prime}}\right)=4$, for all $m^{\prime}, n^{\prime} \in \mathbf{N}$. Now, since there exists a star $S_{4} \subset P_{m} \times C_{5}$, then $\lambda_{1}^{1}\left(P_{m} \times C_{5}\right) \geq 5$.

The last theorem clearly yields the next corrolary.

Corollary 4.11. For all $m \geq 3, n^{\prime} \in \mathbf{N}, \lambda_{1}^{1}\left(P_{m} \times C_{5 n^{\prime}}\right)=4$.

Lemma 4.12. Suppose $G^{\prime}$ is a connected component of $P_{3} \times P_{n}, n \geq 9$, such that $u_{i} v_{j}, u_{i} v_{k} \in V\left(G^{\prime}\right)$. If $d\left(u_{i} v_{j}, u_{i} v_{k}\right)=8$, then $l\left(u_{i} v_{j}\right) \neq l\left(u_{i} v_{k}\right)$.

Proof. Suppose $\alpha_{j}, \alpha_{k} \in[4]$ and $\alpha_{j}=l\left(u_{1} v_{j}\right), \alpha_{k}=l\left(u_{1} v_{k}\right)$, while $d\left(u_{1} v_{j}, u_{1} v_{k}\right)=8$. The next vertex, according to Lemmas 4.1 and 4.2 , that $\alpha_{j}$ labels is either $u_{0} v_{j+3}$ and $u_{2} v_{j+3}$. Now, since $d\left(u_{0} v_{j+3}, u_{1} v_{k}\right)=5$, then by Lemma $4.1, \alpha_{j} \neq l\left(u_{1} v_{k}\right)$. Thus, $\alpha_{k} \neq \alpha_{j}$.

Theorem 4.13. For $m \geq 3, \lambda_{1}^{1}\left(P_{m} \times C_{7}\right)=5$. 
Proof. Suppose $\lambda_{1}^{1}\left(P_{m} \times C_{7}\right)=4$. Clearly from an earlier remark, $P_{m} \times C_{7} \equiv G^{\prime}$ where $G^{\prime}$ is a connected component of $P_{m} \times C_{14}$ Also, $G^{\prime} \equiv G^{\prime \prime}$, where $G^{\prime \prime}$ is the connected component of $P_{m} \times P_{15}$, with $u_{i} v_{0} \equiv u_{i} v_{14}$ for all $i \in[(m-1)(\epsilon)]$. Suppose $\bar{G}$ is a subgraph of $G^{\prime \prime}$ induced by the vertex set $U_{i}, U_{i+1}$ and $U_{i+2}$ such that $u_{i} v_{0} \in U_{i}$, and $u_{i+2} v_{0} \in U_{i+2}$. Let $\left\{\alpha_{i}\right\}_{i=0}^{4}=$ [4] and suppose $\alpha_{0}, \alpha_{1}, \alpha_{2}, \alpha_{3}, \alpha_{4}$, labels $u_{i} v_{0}, u_{i+2} v_{0} u_{i+1} v_{1}, u_{i+2} v_{0} u_{i+2} v_{2}$. Then $l\left(u_{0} v_{14}\right)=\alpha_{0}$ and $l\left(u_{2} v_{14}\right)=\alpha_{1}$. Since $d\left(u_{i+1} v_{1}, u_{i+2} v_{13}\right)=2$, then $l\left(u_{i+1} v_{13}\right) \in\left\{\alpha_{3}, \alpha_{4}\right\}$. Without loss of generality, let $l\left(u_{i} v_{13}\right)=\alpha_{3}$. Then $L\left(u_{0} v_{12}, u_{2} v_{12}\right)=\left\{\alpha_{2}, \alpha_{4}\right\}$. Now, $d\left(u_{i+j} v_{k}, u_{i+1} v_{7}\right)=5$ for all $j \in\{0,2\}$, $k \in\{2,12\}$ Thus, by Lemma 4.1, $l\left(u_{i+1} v_{7}\right) \in A=\left\{\alpha_{2}, \alpha_{3}, \alpha_{4}\right\}$. Also, by the reason of distance, $l\left(u_{i+1} v_{3}\right) \in A$. thus, $l\left(u_{i+1} v_{3}\right)$ is either $\alpha_{0}$ or $\alpha_{1}$. Again without loss of generality, suppose $l\left(u_{i+1} v_{3}\right)=\alpha_{0}$. By Lemma 4.2, $l\left(u_{i+1} v_{7}\right) \neq \alpha_{0}$. Thus, $l\left(u_{i+1} v_{7}\right)=\alpha_{1}$. Since $l\left(u_{i+1} v_{7}\right)=\alpha_{1}$, then $l\left(u_{i+1} v_{11}\right) \neq \alpha_{1}$. therefore, $l\left(u_{i+1} v_{11}\right) \notin\left\{\alpha_{1} \cup A\right\}$ and hence, $l\left(u_{i+1} v_{11}\right)=$ $\alpha_{0}$. But this is a contradiction of Lemma 4.12 since $d\left(u_{i+1} v_{3}, u_{i+1} v+11\right)=8$ and it is assumed that $\lambda_{1}^{1}\left(P_{m} \times C_{7}\right)=4$. Thus, $\lambda_{1}^{1}\left(P_{m} \times C_{7}\right) \geq 5$. Conversely, for each $i \in[m-1],\left|V_{i}\right|=7$, where $V_{i} \subset V\left(G^{\prime}\right)$. Therefore, suppose $\left|L\left(V_{i}\right)\right|=6$, then there exists a pair $v_{1}, v_{2} \in V_{i}$ such that $l\left(v_{i}\right)=l\left(v_{2}\right)=\alpha_{k}$ for some $\alpha_{k} \in[5]$. Now, set $u_{1}=u_{i} v_{j}$ and $u_{2}=u_{i} v_{j+4}$ such that $d\left(u_{i} v_{j}, v_{j+4}\right) d\left(u_{1}, u_{2}\right)=4$. Let $\bar{V}_{1}=V_{i} \backslash\left\{u_{i} v_{j}\right\}$. Set $\alpha_{j}=l\left(u_{k} v_{l}\right)=$ $l\left(u_{k+1} v_{l+3}\right)$ for all $\mathrm{u} u_{k} v_{j} \in \bar{V}_{1}$. Now, there exists $u_{3}=u_{k+3} v_{j+3} \in V_{i+1}$ such that $u_{3}$ is not yet labeled. Let $u_{4}=u_{k+1} v_{j-1}$ and set $l\left(u_{k+1} v_{j-1}\right)=$ $l\left(u_{k+1} v_{j+3}\right)$. Obviously, $d\left(u_{3}, u_{4}\right)=4$ and $u_{3}, u_{4} \in V_{i+1}$. Repeat the above scheme between $V_{i+1}$ and $V_{i+2}, V_{i+2}$ and $V_{i+3}, \ldots, V_{m-2}, V_{m-1}$. Thus $\lambda_{1}^{1}\left(P_{m} \times C_{7}\right) \leq 5$ and then the equality follows.

The proof of the next results follow the last theorem and some remarks made earlier.

Corollary 4.14. For $m \geq 3, \lambda_{1}^{1}\left(P_{m} \times C_{14}\right)=5$.

Theorem 4.15. Let $m \geq 3$. Then $\lambda_{1}^{1}\left(P_{m} \times C_{8}\right)=5$.

Proof. That $\lambda_{1}^{1}\left(P_{m} \times C_{8}\right) \geq 5$ follows from Lemma 4.12 and $\lambda_{1}^{1}\left(P_{m} \times\right.$ $\left.C_{8}\right) \leq 5$ follows from repeating the $L(1,1)$-labeling of $P_{m} \times C_{4}$. 


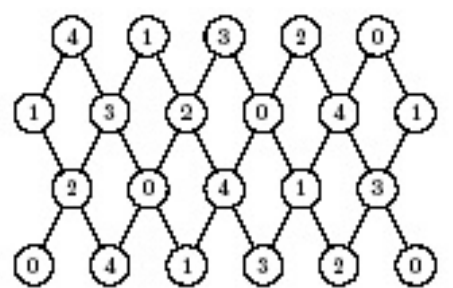

(a) $P_{4} \times C_{10}$

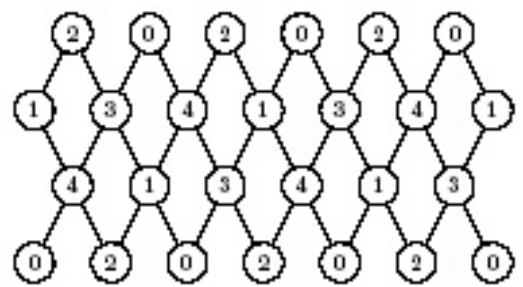

(b) $P_{4} \times C_{12}$

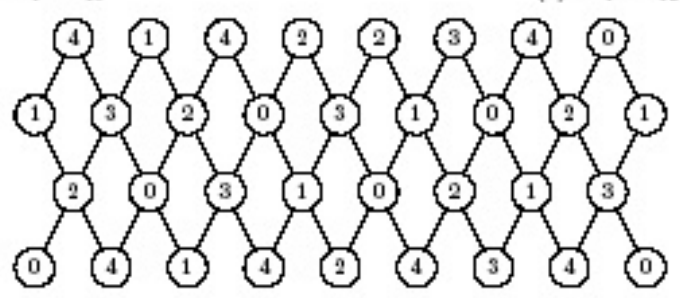

(c) $P_{4} \times C_{16}$

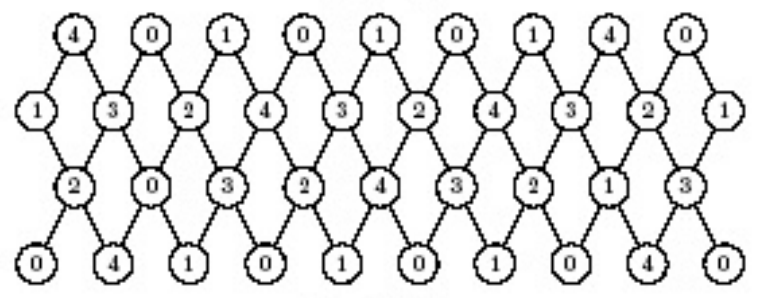

(d) $P_{4} \times C_{13}$

Fig. $14-L(1,1)$-Labeling of $P_{4} \times C_{n}, n-10,12,16,18$

Theorem 4.16. Given that $n \geq 9, n \neq 14$, then $\lambda_{1}^{1}\left(P_{4} \times C_{n}\right)=4$.

Proof. From (b), (c),(d) of Fig. 1, we notice that $\lambda_{1}^{1}\left(P_{4} \times C_{n^{\prime}}\right)=4$, for all $m^{\prime} \in\{12,16,18\}$.

Now, by combining each of (b),(c),(d) with (a), we see that $\lambda_{1}^{1}\left(P_{m} \times\right.$ $\left.C_{n^{\prime}+10}\right)=4$, for each $n^{\prime} \in\{12,16,18\}$. Therefore, $\lambda_{1}^{1}\left(P_{4} \times C_{k m^{\prime}+p}\right)=4$ $\forall k \geq 0$ and $p \in\{0,10\}$. Thus by an earlier remark, $\lambda_{1}^{1}\left(P_{4} \times C_{n}\right)=4$ for all $n \geq 9, n \neq 14$. 
Corollary 4.17. Given that $n \geq 9, n \neq 14$, and that $m \in\{3,4\}$ then $\lambda_{1}^{1}\left(P_{m} \times C_{n}\right)=4$.

Theorem 4.18. For $m \geq 3, \lambda_{1}^{1}\left(P_{m} \times C_{14}\right)=4$.

Proof. It follows directly from Remark 4.4 (iii) and Theorem 4.13.

Next, we derive the general lower bound for the $L(1,1)$ - labelling of $P_{m} \times C_{n}$, where $m \geq 5, n \not \equiv 0 \bmod 5$. That $\lambda_{1}^{1}\left(P_{m} \times C_{n}\right)=4$, where $m, n$ are both multiples of 5 , has already been established. We need the next lemma to prove the theorem that follows.

Lemma 4.19. If $\lambda_{1}^{1}\left(P_{m} \times C_{n}\right)=4$ for $n \not \equiv 0 \bmod 5, n \geq 9$. Then, for all $V_{j} \subset V\left(P_{m} \times C_{n}\right), 0 \leq j \leq n-2$, there exist $v_{a}, v_{b} \in V_{j}$, such that $l\left(v_{a}\right)=l\left(v_{b}\right)$ and $d\left(v_{a}, v_{b}\right)=6$.

Proof. Let $G=P_{m} \times C_{n}$. Suppose, without loss of generality, that $n$ is even since by Remark 4.4 (iii), if $n$ is odd then $G$ is equivalent to one of the two components of $P_{m} \times C_{2 n}$. Let $G^{\prime}$ be the connected component of $G$. Let $V_{j}^{\prime} \subset V\left(G^{\prime}\right)$ such that $V_{j}^{\prime} \subset V_{j}$. Let $v_{a} \in V_{j}^{\prime}$ such that $l\left(v_{a}\right)=\alpha_{k} \in[4]$. Since $n$ is not a multiple of 5 , and $n \geq 9$, then $\left|V_{j}^{\prime}\right|=\frac{n}{2}>5$. Since $\lambda_{1}^{1}(G)=4$, then there exists at least some vertex $v_{b} \in V_{j}^{\prime}$ such that $l\left(v_{b}\right)=\alpha_{k}$. By the definition of $L(1,1)$ - labeling, $d\left(v_{a}, v_{b}\right) \neq 2$. Likewise by Lemmas 4.2 and $4.12, d\left(v_{a}, v_{b}\right) \notin\{4,8\}$ thus, $d\left(v_{a}, v_{b}\right)=6$.

Theorem 4.20. Let $m \geq 5, n \not \equiv 0 \bmod 5$ and $n \geq 9$. Then, $\lambda_{1}^{1}\left(P_{m} \times C_{n}\right) \geq$ 5.

Proof. Let $m \geq 5, n \not \equiv 0 \bmod 5$ and $n \geq 9$. Suppose $\lambda_{1}^{1}\left(P_{m} \times C_{n}\right)=4$. Let $G=P_{m} \times C_{n}$. Suppose $n$ is even. Then there exists $G^{\prime}$, a connected component of $P_{m} \times C_{n}$. (If $n$ is odd, we know from an earlier result that $G$ is a connected component of $P_{m} \times C_{2 n}$.) We defined an arbitrary vertex set $V\left(G^{\prime \prime}\right)=\left\{u_{i} v_{j}, u_{i} v_{j+2}, u_{i+1} v_{j+1}, u_{i+2} v_{j}, u_{i+2} v_{j+2}, u_{i+3} v_{j+1}, u_{i+4} v_{j}, u_{i+4} v_{j+2}\right\}$, with $V\left(G^{\prime \prime}\right) \subset V\left(G^{\prime}\right)$. Clearly, $V\left(G^{\prime \prime}\right)$ induces a subgraph $G^{\prime \prime}$ of $G^{\prime}$ such that $G^{\prime \prime}=S_{4}^{\prime} \cup S_{4}^{\prime \prime}$ where $S_{4}^{\prime}, S_{4}^{\prime \prime}$ are stars with $V\left(S_{4}^{\prime}\right)=\left\{u_{i} v_{j}, u_{i} v_{j+2}, u_{i+1} v_{j+1}, u_{i+2} v_{j}, u_{i+2} v_{j+2},\right\}$ and $S_{4}^{\prime \prime}=u_{i+2} v_{j}, u_{i+2} v_{j+2}, u_{i+3} v_{j+i}, u_{i+4} v_{j}, u_{i+4} v_{j+2}$ respectively. Now, by 4.19 above, for all $V_{i} \subset V\left(G^{\prime}\right), 0 \leq i \leq m-2$ there exist at least a vertex pair 
$v_{a}, v_{b} \in V_{i}$ such that for some $\alpha_{i} \in L\left(V_{i}\right) \subseteq[4], l\left(v_{a}\right)=l\left(v_{b}\right)=\alpha_{i}$ and $d\left(v_{a}, v_{b}\right)=6$.

Suppose $u_{i+2} v_{j-2}, u_{i+2} v_{j+4} \in V_{i+2}$ such that $l\left(u_{i+2} v_{j-2}\right)=l\left(u_{i+2} v_{j+4}\right)=$ $\alpha_{i}$.

There exist vertices $u_{i+1} v_{j+1} \in V_{i+1}$ and $u_{i+3} v_{j+1} \in V_{i+3}$.

By Lemma 4.1, $l\left(u_{i+1} v_{j+1}\right)=\alpha_{i}$ or $l\left(u_{i+3} v_{j+1}\right)=\alpha_{i}$. Suppose

$l\left(u_{i+1} v_{j+1}\right)=\alpha_{i}$, then $d\left(u_{a}, u_{b}\right) \leq 2$ for any $u_{a} \in V\left(S_{4}^{\prime \prime}\right)$ and $u_{b} \in\left\{u_{i+1} v_{j+1}, u_{i+2} v_{j-2}, u_{i+2} v_{j+4}\right\}$.

Thus there is no such vertex as $u_{a} \in S_{4}^{\prime}$ such that $l\left(u_{a}\right)=\alpha_{i} \in V\left(S_{4}^{\prime}\right)$. Likewise, $d\left(u_{a}^{\prime}, u_{b}\right) \leq 2$ for any $u_{a}^{\prime} \in V\left(S_{5}^{\prime}\right)$ and $u_{b} \in\left\{u_{i+3} v_{j+1}, u_{i+2} v_{j-2}, u_{i+2} v_{j+4}\right\}$.

Thus, there exists no vertex $u_{a}^{\prime} \in V\left(S_{5}^{\prime}\right)$, such that $l\left(u_{a}^{\prime}\right)=\alpha_{j} \in[4]$ and therefore, a contradiction.

By the result obtained in Theorem 4.20, we see that the $\lambda_{1}^{1}\left(P_{m} \times C_{n}\right) \geq 5$ for all $m \geq 5$ and $n \geq 9$, where $n$ is not a multiple of 5 . In the subsequent results, we obtain the $\lambda_{1}^{1}$-number for the remaining $P_{m} \times C_{n}$ graphs. 

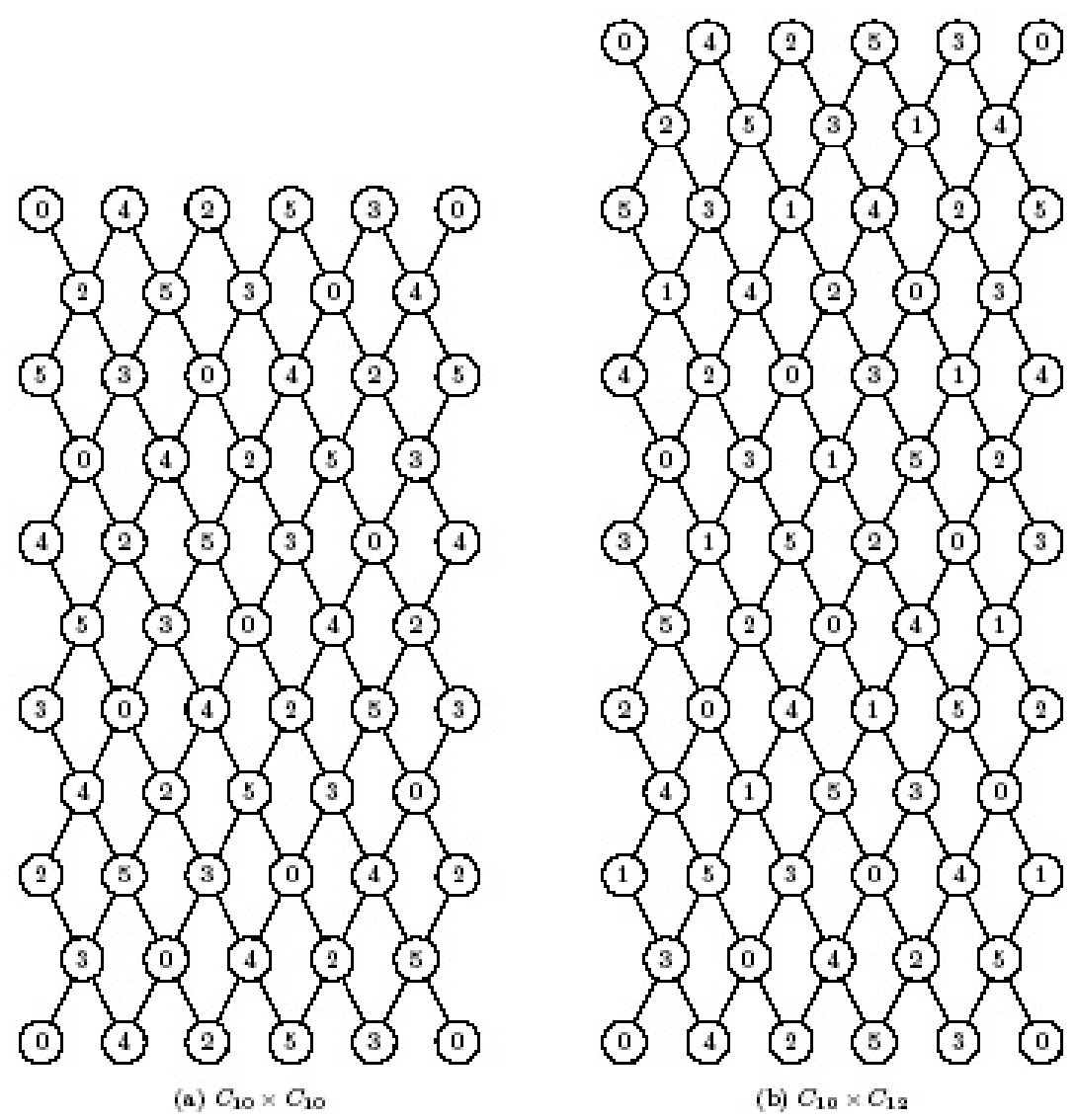

Fig. $25-L(1,1)$-Labeling of $C_{10} \times C_{n}, m-10,12$ 

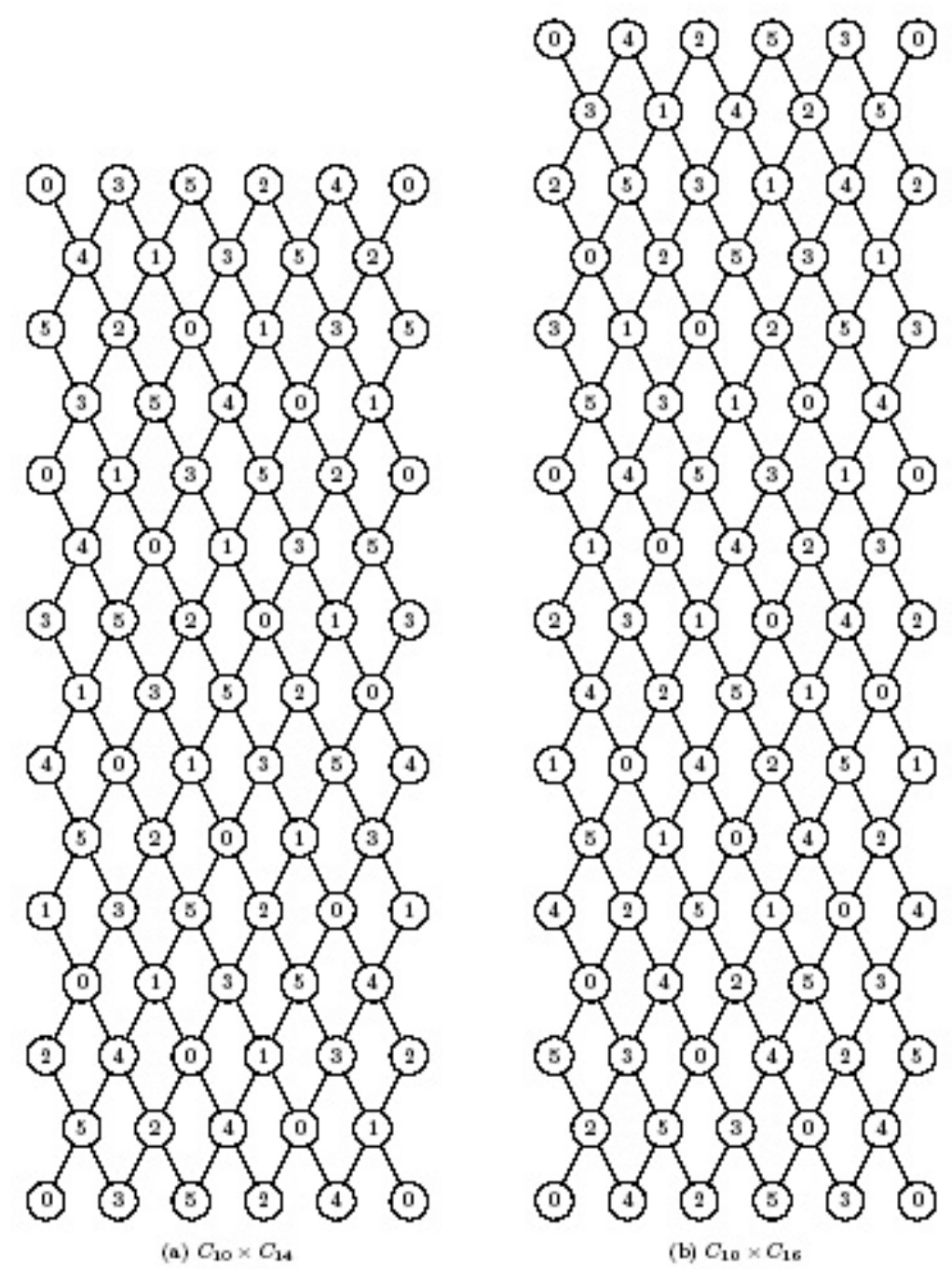


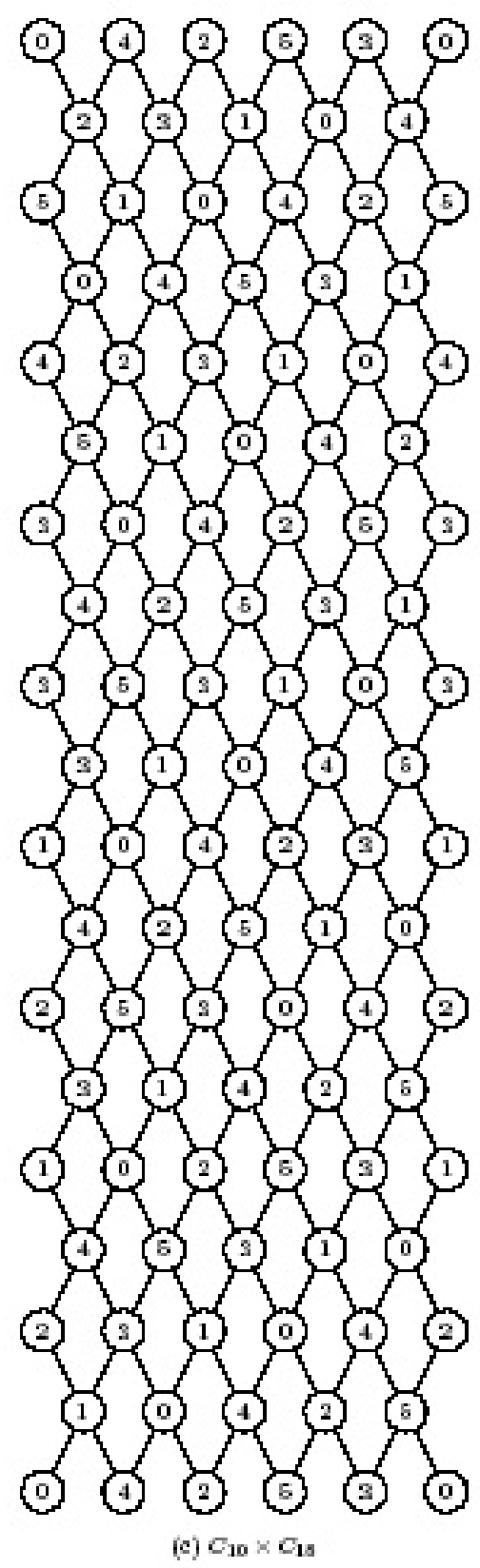

Fle. $35-(L(1,1))$ Labeling of $C_{10} \times C_{n}, n=14,16,18$ 
Theorem 4.21. Let $k \in A$. For all $k, m^{\prime}, n^{\prime}, \lambda_{1}^{1}\left(C_{10 m^{\prime}} \times C_{k+10 n^{\prime}}\right)=$ 5 , where $m^{\prime}$ is any positive integer, $n^{\prime}$ a non-negative integer and $A=$ $\{12,14,16,18\}$.

Proof. The result follows by combining the 5-labeling of $C_{10} \times C_{10 n^{\prime}}$ which is obtainable from $n^{\prime}$-times repeat of Fig.5 a, with the 5 -labeling of $C_{10} \times C_{12}, C_{10} \times C_{14}, C_{10} \times C_{16}$ and $C_{10} \times C_{18}$ in Fig.5 b and of Fig. 3 a, b and c respectively along with $C_{n}$ and then $m^{\prime}$-copy the resultant graph along with $C_{m}$.

Corollary 4.22. For all $P_{m} \times C_{n}$, where $m \geq 5$ and $n \geq 6, n \not \equiv 0 \bmod 5$ then $\lambda_{1}^{1}\left(P_{m} \times C_{m}\right)=5$.

Proof. Let $h$ be a positive even integer with $h \geq 12$. Let $k \in A=$ $\{12,14,16,18\}$. Then, for all $h, h \equiv 0 \bmod k+10 n^{\prime}$ for some $k \in A$. The result thus follows from Remarks 4.4 (iii) and (iv) and the fact that $P_{m} \times C_{n} \subset P_{10 m} \times C_{n}$.

\section{Conclusion}

The following summarizes the results obtained in this work:

For $G=P_{m} \times P_{n}$ :

\begin{tabular}{|c|c|c|}
\hline$m$ & $n$ & $\lambda_{1}^{1}\left(P_{m} \times P_{n}\right)$ \\
\hline 2 & 2 & 1 \\
\hline$\geq 3$ & 2 & 2 \\
\hline$\geq 3$ & $\geq 3$ & 4 \\
\hline
\end{tabular}

For $G=P_{m} \times C_{n}$ :

\begin{tabular}{|c|c|c|}
\hline$m$ & $n$ & $\lambda_{1}^{1}\left(P_{m} \times C_{n}\right)$ \\
\hline 2 & $\equiv 0 \bmod 3$ & 2 \\
\hline 2 & $\not \equiv 0 \bmod 3$ & 3 \\
\hline$\geq 3$ & $\in\{3,4,6,7,8,14\}$ & 5 \\
\hline$\geq 3$ & $\equiv 0 \bmod 5$ & 4 \\
\hline 3,4 & $\geq 9, \neq 14$ & 4 \\
\hline$\geq 5$ & $\geq 9, \not \equiv 0 \bmod 5$ & 5 \\
\hline
\end{tabular}




\section{References}

[1] Bertossi, A. A. and Bonuccelli, M. A. Code Assignment for Hidden Terminal Interference Avoidance in multiloop Pocket Radio Networks. IEEE/ACM Trans. on Networking 3,(4), pp. 441-449, (1995).

[2] Berttiti, R., Bertossi, A. A. and Bonuccelli, M. A. Assigning Codes in Wireless networks: Bounds and Scaling Properties. Wirel. Netw., 5, pp. 441-449, (1999).

[3] Calamonerri, T. The $L(h, k)$-Labeling Problem: A Updated Survey and Annoted Bibliography. The Computer Journal 54(8), pp.1344 $1371,(2011)$.

[4] Calamonerri, T., Pelc, A., and Petreschi, R. Labeling Trees with a Condition at Distance Two. Discrete Math. 306, pp. 1534-1539, (2006).

[5] Chang, G. J. and Kuo, D. The $L(2,1)$-Labeling of Graphs SIAM J. Discrete math. 9, pp. 309-316, (1996).

[6] Chang, G. J., Ke, W.-T, Kuo, D. A., Liu, D. D.-F and Yeh, R. K. $(d, 1)$ - Labeling of Graphs. Discrete Math. 220, pp. 57-66, (2000).

[7] Chiang,S.-H and Yan, J.-H. On $L(d, 1)$-labeling of Cartesian product of a cycle and a path. Discrete App. Math 156, pp. 2867-2881, (2008).

[8] Georges, J. P, and Mauro, D.W. Generalized Vertex Labeling with a Condition at Distance Two. Congr. Numer., 109, pp. 141-159, (1995).

[9] Georges, J. P, Mauro, D. W. and Stein, M. I. Labeling Products of Complete Graphs with a Condition at Distance Two. SIAM J. Discrete Math. 14, pp. 28-35, (2000).

[10] Georges, J. P, Mauro, D. W. On Regular Graphs Optimally labeled with a Condition at Distance Two. SIAM J. Discrete Math. 17 (2), pp. 320-331, (2003).

[11] Goncalves, D. $L(p-1)$-Labellings of Graphs. Discrete Math. 308, pp. 1405-1414, (2008).

[12] Gravier, S., Klavzer, S. and Mollard M., Codes and L(2,1)-Labeling in Sierpinski Graphs. Taiwan. J. math. 4, pp. 671-681, (2004). 
[13] Griggs, J. R., Yeh, R. K. Labeling Graph with a condition at Distance Two. SIAM J. Discrete Math. 5, pp. 586-595, (1992).

[14] Hale, W. K. Frequency Assignment: Theory and Application. Proc. IEEE 68, pp. 1497-1514, (1980).

[15] Jha, P. K, Klavzer, S. and Vessel, A. L(2,1)-Labeling of Direct Product of Paths and Cycles. Discrete Applied Math. 145 (2), pp. 141-159, (2005).

[16] Kral', D. and Skrekovski, R. A. Theorem About the Channel Assignment J. Discrete Math. 16 (3), pp. 426-437, (2003).

[17] Kuo, D. and Yan, J.-H. On $L(2,1)$-Labeling of Cartesian Product of Two Cycles. Discrete Math. 283, pp. 137-144, (2004).

[18] Sakai, T. D. Chordal Graphs with a Condition at Distance Two. SIAM J. Discrete Math. 7, pp. 133-140, (1994).

[19] Schwartz, C. and Sakai, T. D. L(2,1)- Labeling of Product of Two Cycles. Discrete Applied Math. 154, pp. 1522-1540, (2006).

[20] Sopena, E., Wu, J. Coloring the square of the Cartesian product of two Cycles Graphs with a Condition at Distance Two. Discrete Math. 310, pp. 2327-2333, (2010).

[21] Wensong, D. and Peter C.,L. Distance Two Labeling and Direct Product of Graphs. Discrete Math. 308, pp. 3805-3815, (2008).

[22] Weichsel, P. M. The Kronecker Product of Graphs. Proc. Amer. Math. Soc. 13 1962; $47-62$

[23] Whittlessey, M. A, Georges, J. P. and Mauro, D. W. On the $\lambda$-number of $Q_{n}$ and Related Graphs. SIAM J. Discrete Math. 8, pp. 499-506, (1995).

\section{Deborah Olayide Ajayi}

Department of Mathematics

University of Ibadan,

Ibadan,

Nigeria

e-mail : olayide.ajayi@mail.ui.edu.ng 
and

Tayo Charles Adefokun

Department of Computer and Mathematical Sciences, Crawford University,

Nigeria

e-mail : tayoadefokun@crawforduniversity.edu.ng 